\title{
Gamma-H2AX biodosimetry for use in large scale radiation incidents: comparison of a rapid ' 96 well lyse/fix' protocol with a routine method
}

Following a radiation incident, preliminary dose estimates made by $\gamma-\mathrm{H} 2 \mathrm{AX}$ foci analysis can supplement the early triage of casualties based on clinical symptoms. Sample processing time is important when many individuals need to be rapidly assessed. A protocol was therefore developed for high sample throughput that requires less than $0.1 \mathrm{ml}$ blood, thus potentially enabling finger prick sampling. The technique combines red blood cell lysis and leukocyte fixation in one step on a 96 well plate, in contrast to the routine protocol, where lymphocytes in larger blood volumes are typically separated by Ficoll density gradient centrifugation with subsequent washing and fixation steps. The rapid '96 well lyse/fix' method reduced the estimated sample processing time for 96 samples to about $4 \mathrm{~h}$ compared to $15 \mathrm{~h}$ using the routine protocol. However, scoring 20 cells in 96 samples prepared by the rapid protocol took longer than for the routine method (3.1 versus $1.5 \mathrm{~h}$ at zero dose; 7.0 versus $6.1 \mathrm{~h}$ for irradiated samples). Similar foci yields were scored for both protocols and consistent dose estimates were obtained for samples exposed to $0,0.2,0.6,1.1,1.2$, 2.1 and $4.3 \mathrm{~Gy}$ of $250 \mathrm{kVp}$ X-rays at $0.5 \mathrm{~Gy} / \mathrm{min}$ and incubated for $2 \mathrm{~h}$. Linear regression coefficients were $0.87 \pm 0.06\left(R^{2}=97.6 \%\right)$ and $0.85 \pm 0.05\left(R^{2}=98.3 \%\right)$ for estimated versus actual doses for the routine and lyse/fix method, respectively. The lyse/fix protocol can therefore facilitate high throughput processing for $\gamma-\mathrm{H} 2 \mathrm{AX}$ biodosimetry for use in large scale radiation incidents, at the cost of somewhat longer foci scoring times. 
1 Jayne Moquet, Stephen Barnard and Kai Rothkamm*

2 Public Health England, Centre for Radiation Chemical and Environmental Hazards, Chilton,

3 Didcot, Oxfordshire OX11 0RQ, UK

4 *Corresponding author: Kai Rothkamm, PHE CRCE, Chilton, Didcot, Oxfordshire OX11 0RQ, 5 UK. Tel. +44 1235 831600; email: kai.rothkamm@phe.gov.uk 
7 The $\gamma$-H2AX assay has been shown to be a reliable and sensitive indicator of radiation-induced 8 DNA double-strand breaks (Rothkamm \& Löbrich, 2003; Ivashkevich et al. 2012). In addition, 9 several ex vivo studies (Horn, Barnard \& Rothkamm, 2011; Horn \& Rothkamm, 2011; Roch10 Lefevre et al. 2010; Mandina et al. 2011) have shown the potential of the $\gamma$-H2AX assay as a useful biodosimetry tool from hours to $\sim 3$ days post exposure. The $\gamma$-H2AX assay can produce dose estimates within a few hours of receiving a blood sample (Rothkamm et al. 2013a; Ainsbury et al. 2013) making it a good candidate for high through-put biodosimetry in the case of a mass casualty event. One option has been to develop fully automated systems such as the RABIT system (Garty et al. 2010; Turner et al. 2011) capable of processing several thousand samples a day. A drawback of this approach is that it requires highly sophisticated robotic equipment and tailored process control software, which may be unavailable to some laboratories. An alternative option to increase through-put is to have an assistance network of laboratories, such as those involved in the European funded MULTIBIODOSE and RENEB projects, and to reduce the processing time required for the $\gamma-\mathrm{H} 2 \mathrm{AX}$ assay using routinely available equipment.

Here a method is presented for the assessment of $\gamma$-H2AX foci for biological dosimetry, that uses a lyse/fix buffer to lyse red blood cells and fix leukocytes in one step from small 'finger prick'sized blood samples. Potentially, the 96-well plate-compatible lyse/fix method would be a faster and more scalable technique for high sample through-put compared to the routine protocol used to process samples for $\gamma-\mathrm{H} 2 \mathrm{AX}$ foci scoring, which involves the isolation of lymphocytes from at least $2 \mathrm{~mL}$ of blood using Ficoll density gradient centrifugation with subsequent washing and fixation steps.

\section{MATERIALS AND METHODS}

\section{Blood sampling and irradiation}

Heparinised venous blood was taken with written informed consent and the ethical approval of the Berkshire research ethics committee (Ref 09/H0505/87) from 17 healthy donors. Whole blood was placed into $15 \mathrm{ml}$ centrifuge tubes, positioned inside a $22 \mathrm{~mm}$ polystyrene block with $8 \mathrm{~mm}$ Perspex, and sham-exposed or exposed to 0.5 or $1.0 \mathrm{~Gy}$ of $250 \mathrm{kVp} \mathrm{x}$-rays (with a half-value layer of $\mathrm{Cu} / \mathrm{Al}$ filtration) at a dose rate of $0.5 \mathrm{~Gy} /$ minute (min). Dosimetry was performed with a calibrated reference ionisation chamber for the exact exposure setup used. Exposures were always monitored using a calibrated UNIDOS E electrometer and 'in-beam' monitor ionisation chamber (all from PTW, Germany). Spatial dose uniformity was checked using Gafchromic EBT2 films (Vertec Scientific Ltd, UK). Following irradiation, the blood was held at $37{ }^{\circ} \mathrm{C}$ for 0.5 or 1 hour to simulate in vivo repair. Samples were then processed for the assessment of $\gamma$ $\mathrm{H} 2 \mathrm{AX}$ foci by the routine histopaque or rapid lyse/fix method. A record was made of the time taken for each step in the process. To test the suitability of the lyse /fix method for biological dosimetry, samples were exposed to x-ray doses up to $4.3 \mathrm{~Gy}$ and incubated for 2 hours at $37^{\circ} \mathrm{C}$. Coded samples were processed using both methods, scored and used to produce estimates of dose..

\section{Rapid 96 well lyse/fix method}

46 Aliquots of $90 \mu \mathrm{L}$ whole blood were placed into a 96 deep well plate (\#FB58005; Fisher

47 Scientific, UK). To each well, 1x lyse/fix buffer (\#558049; BD Biosciences, UK) pre-warmed to

$4837^{\circ} \mathrm{C}$ was added to give a dilution of $1: 20$, thoroughly mixed and incubated at $37^{\circ} \mathrm{C}$ for $14 \mathrm{~min}$. 
After centrifugation at $250 \mathrm{~g}$ for 5 min the supernatant was removed using an 8 channel aspirator. Following two washes in cold phosphate buffered saline (PBS) the cell pellet was re-suspended in $\sim 50 \mu \mathrm{L}$ of PBS. An aliquot of $25 \mu \mathrm{L}$ per sample was spotted onto a teflon/silane coated 14-well slide (Tekdon Incorporated, USA) and the cells were allowed to adhere for $\sim 15 \mathrm{~min}$. When the slides were almost dry they were placed in a moist camber and incubated in $0.5 \%$ Triton-X (Sigma, UK) in PBS for $5 \mathrm{~min}$. The slides were then drained and incubated for $10 \mathrm{~min}$ in blocking solution (BS), containing 1\% bovine serum albumin (Sigma, UK) in PBS. Once any excess liquid had been removed, $100 \mu \mathrm{L}$ of mouse $\gamma$-H2AX antibody (\#613402; BioLegend, UK), diluted 1:500 in BS, was applied to each slide. After incubating the slides in the dark for $40 \mathrm{~min}$ at room temperature they were washed 3 times in BS. Slides were then incubated in the dark with $100 \mu \mathrm{L}$ of goat anti-mouse AlexaFlour 488 secondary antibody (Abcam, UK), diluted 1:500 in $\mathrm{BS}$, together with $0.2 \mu \mathrm{g} / \mathrm{mL}$ 4',6-diamidino-2-phenylindole (DAPI) for $30 \mathrm{~min}$ at room temperature. Finally the slides were washed three times in PBS and mounted in Vectashield antifade solution (Vector Laboratories, UK). A detailed description of the lyse/fix protocol, sample requirements and materials can be found in Supplementary material: A Rapid Protocol for $\gamma$ H2AX Processing.

\section{Routine histopaque method}

The histopaque method has been described in detail by Rothkamm et al (2013b). In brief, $2 \mathrm{~mL}$ of whole blood was mixed 50:50 with PBS and layered onto $4 \mathrm{~mL}$ Histopaque 1077 (Sigma, UK) in $15 \mathrm{ml}$ conical centrifuge tubes. After centrifugation at $1200 \mathrm{~g}$ for $5 \mathrm{~min}$, the buffy coat layer was washed twice in PBS and the cell pellet re-suspended in $\sim 0.1 \mathrm{~mL}$ of PBS. $10 \mu \mathrm{L}$ of the cell suspension was spotted onto a multi-well slide. Cells were allowed to adhere to the slide for $\sim 15$ min and then fixed in 2\% formaldehyde in PBS for $5 \mathrm{~min}$, followed by $5 \mathrm{~min}$ in $0.25 \%$ Triton$\mathrm{X} 100+0.1 \%$ glycine in PBS. At this stage the blocking step and the immuno-staining was carried out in the same manner as the lyse/fix method described above.

\section{Manual scoring of $\gamma-\mathrm{H} 2 \mathrm{AX}$ foci}

All the slides were examined at x600 magnification using a Nikon Optiphot 2 fluorescence microscope, equipped with separate filters for DAPI and fluorescein isothiocyanate (FITC). Manual scoring was timed for three unirradiated and three $1 \mathrm{~Gy} / 1 \mathrm{~h}$ incubated samples on slides produced by both processing methods. A total of 50 lymphocytes were scored per sample and the time taken to do this was recorded every ten cells. To ensure the lyse/fix processing itself did not affect foci levels, samples irradiated at $0.5 \mathrm{~Gy} /$ repair time 30 minutes and prepared using both protocols were used to assess the number of foci per cell seen in a panel of 17 donors. 50 lymphocytes were scored in each of the reference samples and the foci numbers were used to adjust the calibration coefficients and the associated standard errors (Rothkamm et al. 2013b) of the laboratory's calibration curve (Horn, Barnard \& Rothkamm, 2011). Blood dose estimates for the unknown samples were produced by scoring up to 50 lymphocytes or 200 foci per sample.

\section{Automated scoring of $\gamma-\mathrm{H} 2 \mathrm{AX}$ foci}

The performance of automated scoring was also assessed on samples processed by the two methods. For each processing method and the two irradiation conditions, 10 fields of view were scored automatically. Maximum projection images of seven $\mathrm{z}$ planes at $1 \mu \mathrm{m}$ step size were captured for $\gamma-\mathrm{H} 2 \mathrm{AX}$ and DAPI staining (only one central plane imaged) using a $\mathrm{x} 40$ objective. Foci analysis was performed with the TRI2 program, which contains batch processing functionality and automatic sorting of images. The method used for foci counting and analysis was the Compact Hough and Radial Map (CHARM) algorithm aimed at faint and ill-defined 
94 shapes (Barber et al. 2007). The foci analysis software has been fully described in Rothkamm et 95 al. (2012).

96 Statistical analysis

97 Mean foci yields and standard deviations were calculated. The Student's t-test was performed to 98 compare foci yields. Linear regressions were performed using Minitab $^{\mathrm{TM}} 15$ to assess the 99 accuracy of dose estimations using the two protocols. Constant coefficients were insignificant 100 ( $>>0.4$; t-test) for all regressions. Estimated slope coefficients are reported together with their 101 associated standard errors.

102

103

104

105

106

107

108

109

110

111

112

113

114

115

116

117

118

119

120

121

122

123

124

125

126

127

128

129

130

131

132

133

134

135

136

\section{RESULTS AND DISSCUSSION}

The time taken to process 16 samples using the lyse/fix protocol and 8 samples by the histopaque method is very similar, $179.5 \mathrm{~min}$ and $171 \mathrm{~min}$ respectively - see Figure 1 which also shows the individual protocol steps. The time for each step in both protocols has been recorded and an estimate made of the timings for handling of 96 samples. Scaling up the timings for preparing 96 samples by the lyse/fix or histopaque method gives an increase in the estimated processing time of a factor of $\sim 1.4$ (256 min total) and $\sim 2.5$ (439 min total) respectively. Most of the difference between the two protocols is the time taken to process the samples through the first 3 steps. Provided that multi-channel pipettes and aspirators for liquid handling are used, one person could complete the first three steps of the lyse/fix protocol for 96 samples in $72 \mathrm{~min}$. If one person were to process 96 samples by the histopaque method up to and including step 3 this would take more than three times as long (226 min). In reality, given the long processing time required for one person to get 96 samples to step 4 of the histopaque protocol, which may adversely affect the blood separation or lower foci levels, either several persons would need to work together or the samples be divided into batches. Assuming four batches of 24 samples each, the histopaque processing time for 96 samples would be 880 minutes $(14.7 \mathrm{~h})$ for one operator (Figure 1).

Usually in the routine histopaque protocol cell suspensions are spread onto individual slides, but for a large number of samples this would be time consuming. For the purposes of this comparison, cells prepared by both techniques were spotted onto 14-well slides. The time taken to complete steps 5 to 11 would be similar for both protocols, 169 (lyse/fix) and $198 \mathrm{~min}$ (histopaque). Multi-well slides also enable one person to carry out the protocol after lysis and fixation, as the time between the addition of a solution to the first well and the last is not so great as to adversely affect any step of the staining protocol. However, this is not the case for the histopaque protocol, which requires cells to be fixed after they have been placed on the slide, and this is a time critical step. Again, several persons would be needed to process all the slides together; alternatively they could be stained in batches by one person.

Histopaque separation of whole blood produces a cell suspension containing lymphocytes. In contrast, the lyse/fix protocol produces a cell suspension made up of lymphocytes and other white cell types, which can make foci analysis more challenging. Figure 2 shows images of one field of view of cells processed by the lyse/fix and histopaque methods from irradiated and unirradiated samples. By comparing the images it can be seen that the lyse/fix method gives fewer lymphocytes per field of view (i.e. round cells) and more debris. In addition the foci are not as clearly visible as in samples processed by the histopaque method. Despite differences in the appearance of samples produced by the two methods, manual scoring produced similar foci yields in lymphocytes from a panel of donors exposed to 0.5 Gy x-rays, as shown in Figure 3. 
137 Samples processed by the histopaque and lyse/fix method gave similar average foci counts of $1386.83 \pm 0.80$ and $7.12 \pm 0.51$ respectively $(\mathrm{p}=0.26)$. These values are consistent with previous 139 studies (Horn, Barnard \& Rothkamm, 2011; Chua et al. 2011; Rothkamm et al. 2007).

140 In order to estimate the time required to analyse 96 samples, three slides for each processing 141 method and dose point were scored. For each slide the time taken to score a batch of 10 cells was 142 recorded and then repeated a further 4 times. The average time taken to score each batch of 10 143 cells from the three slides was then used to estimate the time it would take one person to score 20 144 cells from 96 samples. Previous work has demonstrated that scoring 20 cells can produce dose 145 146 estimates that will reliably place samples into the correct exposure categories required for triage (Rothkamm et al. 2013a). As expected the mean time taken to score a 0 Gy sample is less than for a $1 \mathrm{~Gy}$ sample at $1 \mathrm{~h}$ post exposure for both preparation methods. However, for the 0 Gy sample the estimated time taken to score 20 cells in 96 samples prepared by the lyse/fix protocol was about twice as long as for those produced by the histopaque method (Table 1). At the higher dose of $1 \mathrm{~Gy}$ the difference in scoring time between the two processing methods was not as great.

151

Simply comparing the lyse/fix protocol with the histopaque method for processing and scoring all 96 samples suggests the time taken would be similar for both irradiation conditions, $7.4 \mathrm{~h}$ versus $8.8 \mathrm{~h}$ (unirradiated) and $11.3 \mathrm{~h}$ versus $14.4 \mathrm{~h}$ (irradiated) respectively. However, the histopaque protocol requires at least two people to achieve this compared to one for the lyse/fix method. A more realistic comparison involves the 96 samples being split into 4 batches for the histopaque method to be carried out by one person. When this is done the estimated time taken to process and score 96 samples by the histopaque method is $16.2 \mathrm{~h}$ (unirradiated) and $20.8 \mathrm{~h}$ (irradiated). This is approximately twice the time required for sample processing and scoring using the lyse/fix protocol. Furthermore, reagent costs for the histopaque method ( $£ 116 / 96$ samples) are twice as high as those for the lyse/fix protocol ( £57/96 samples).

161 The more challenging analysis procedure required for samples produced by the lyse/fix method

162

163

164

165

166

167

168

169

170

171

172

173

174

175 may be aided by an automated scoring approach. Image analysis software using a form factor parameter would allow the positive discrimination of round lymphocyte nuclei, thereby enriching their number in the analysed cell population (Valente et al. 2011). Here, TRI2 software was used to identify round cell nuclei and score foci automatically (Rothkamm et al. 2012). Figure 4 shows the automated scoring results of 10 fields of view for each processing method and the two irradiation conditions. Automated foci counts are comparable for irradiated samples with averages of $3.49 \pm 0.14$ for lyse/fix and $3.76 \pm 0.06$ for histopaque $(\mathrm{p}=0.84)$, but differ for baseline samples $(0.16 \pm 0.05$ vs. $0.018 \pm 0.004 ; p=0.04)$. Foci counts for irradiated samples were always significantly higher than baseline counts $(p<0.001)$. Importantly, ten times more scorable cells per field of view are seen with the histopaque protocol. The lyse/fix protocol produced 3 false negative fields out of 10 compared to 1 for the histopaque method, likely caused by high background fluorescence due to excessive debris in lyse/fix samples. These results suggest that if automated scoring were to be used to analyse samples, at least 2 (histopaque) and 3 (lyse/fix) fields of view need to be scored and results checked to ensure consistency. Further optimisation of the software may reduce the number of false negative fields.

177 To determine whether the rapid lyse/fix protocol can still provide dose estimates with similar 178 accuracy as the routine histopaque protocol, an intercomparison exercise was performed using 179 uniformly X-irradiated, coded samples. Figure 5 shows that the dose estimates obtained for the 180 two different protocols correlated very well with each other (linear regression slope 1.03 \pm 0.03 ; $181 \mathrm{R}^{2}=99.5 \%$ ). Linear regression of estimated versus true doses produced a slope of $0.87 \pm 0.06$ 
182

183

184

185

186

187

188

189

190

191

192

193

194

195

196

197

198

199

200

201

202

203

204

205

206

207

208

209

210

211

212

213

214

215

216

217

218

219

220

221

222

223

224

$\left(\mathrm{R}^{2}=97.6 \%\right)$ for the histopaque method and $0.85 \pm 0.05\left(\mathrm{R}^{2}=98.3 \%\right)$ for the lyse/fix method. These results demonstrate excellent correlation between the two methods, but a modest systematic bias to under-estimate true doses, illustrating the need for frequent re-calibration of foci-based biodosimetry approaches to compensate any 'drift' in foci detection (Rothkamm et al. 2013b). Given the growing interest in point of care diagnostics, finger prick sampling of capillary blood has been widely tested and confirmed in numerous studies as a suitable alternative to venipuncture. Examples of such studies include a comparison of seroepidemiology of hepatitis B (Bond et al. 1978), CD4 cell counting (MacLennan et al. 2007) or differential leukocyte counting (Hollis et al. 2012). We are not currently aware of any specific comparison of gamma-H2AX induction following irradiation but expect that results for capillary and venipuncture samples should be similar for this endpoint. One exception might be a scenario where blood is taken within seconds (or perhaps up to a few minutes) following a partial body exposure. In such a situation, blood lymphocytes in capillaries may not have had sufficient time to mix completely. However, this could only be of concern in studies where samples are taken immediately following a planned, very short and localised exposure, such as a CT scan (Rothkamm et al. 2007). Naturally, the lyse/fix method presented here would also work with venipuncture samples where it would save blood, money and time that could then be used for other tests.

\section{CONCLUSION}

The lyse/fix protocol enables a high sample throughput, with an estimated processing time for 96 donors of just over 4 hours, using finger prick-sized quantities of blood. This can be achieved by one person, without the need for highly sophisticated equipment. The manual microscope-based analysis of samples produced by the lyse/fix protocol is more challenging, which increases the scoring time, but has no adverse effect on dose estimation. Automated scoring may solve this problem but appears to be more prone to producing false-negative results for samples processed with the lyse/fix protocol compared to the histopaque method. Overall the rapid lyse/fix method allows one person to process and score 96 samples in about half the time taken using the routine protocol.

\section{REFERENCES}

Ainsbury EA, Al-hafidh J, Bajinskis A, Barnard S, Barquinero JF, Beinke C, de Gelder V, Gregoire E, Jaworska A, Lindholm C, Lloyd D, Moquet J, Nylund R, Oestreicher U, RochLefevre S, Rothkamm K, Romm H, Scherthan H, Sommer S, Thierens H, Vandevoorde C, Vral A, Wojcik A. 2013. Inter- and intra-laboratory comparison of a multibiodosimetric approach to triage in a simulated, large scale radiation emergency. International Journal of Radiation Biology doi:10.3109/09553002.2014.868616.

Barber P, Locke R, Pierce G, Rothkamm K, Vojnovic B. 2007. Gamma-H2AX foci counting: image processing and control software for high-content screening. Proceedings of SPIE 6441:64411M-1.

Bond WW, Petersen NJ, Favero MS, Schable CA, Murphy BL, Berquist KR, Maynard JE. 1978. Evaluation of a finger prick blood collection method for the seroepidemiology of hepatitis B. Bulletin of the World Health Organization 56:791-796.

Chua M L, Somaiah N, A'Hern R, Davies S, Gothard L, Yarnold J, Rothkamm K. 2011. Residual DNA and chromosomal damage in ex vivo irradiated blood lymphocytes correlated with late normal tissue response to breast radiotherapy. Radiotherapy and Oncology 99:362-366.

PeerJ reviewing PDF | (v2013:12:1121:1:0:NEW 29 Jan 2014) 
Garty G, Chen Y, Salerno A, Turner H, Zhang J, Lyulko O, Berucci A, Xu Y, Wang H, Simann N, Randers-Pehrson G, Yao Y, Amundson S, Brenner D. 2010. The RABIT: A rapid automated biodosimetry tool for radiological triage. Health Physics 98:209-217.

IAEA. 2011. Cytogenetic dosimetry: applications in preparedness for and response to radiation emergencies. International Atomic Energy Agency, Vienna.

Hollis VS, Holloway JA, Harris S, Spencer D, van Berkel C, Morgan H. 2012. Comparison of venous and capillary differential leukocyte counts using a standard hematology analyzer and a novel microfluidic impedance cytometer. PLoS One 7:e43702.

Horn S, Barnard S, Rothkamm K. 2011. Gamma-H2AX-Based Dose Estimation for Whole and Partial Body Radiation Exposure. PLoS ONE 6:e25113.

Horn S, Rothkamm K. 2011. Candidate protein biomarkers as rapid indicators of radiation exposure. Radiation Measurements 46:903-906.

Ivashkevich A, Redon C, Nakamura A Martin R, Martin O. 2012. Use of the gamma-H2AX assay to monitor DNA damage and repair in translational cancer research. Cancer Letters 327:123133.

MacLennan CA, van Oosterhout JJ, White SA, Drayson MT, Zijlstra EE, Molyneux ME. 2007. Finger-prick blood samples can be used interchangeably with venous samples for CD4 cell counting indicating their potential for use in CD4 rapid tests. AIDS 21:1643-1645.

Mandina T, Roch-Lefevre S, Voisin P, González J, Lamadrid A, Romero I, Garcia O, Voisin P, Roy L. 2011. Dose-response relationship of gamma-H2AX foci induction in human lymphocytes after X-rays exposure. Radiation Measurements 46: 997-999.

Roch-Lefevre S, Mandina T, Voisin P, Gaetan G, Mesa J, Valente M, Bonnesoeur P, Garcia O, Voisin P, Roy L. 2010. Quantification of gamma-H2AX foci in human lymphocytes: a method for biological dosimetry after ionizing radiation exposure. Radiation Research 174:185-194.

Rothkamm K, Balroop S, Shekhdar J, Fernie P, Goh V. 2007. Leukocyte DNA damage following multi-detector row CT: a quantitative biomarker of low level radiation exposure. Radiology 242:244-251.

Rothkamm K, Horn S, Scherthan H, Rößler U, De Amicis A, Barnard S, Kulka U, Lista F, Meineke V, Braselmann H, Beinke C, Abend M. 2013a. Laboratory intercomparison on the gamma-H2AX foci assay. Radiat Res, 180, 149-155.

Rothkamm K, Barnard S, Ainsbury EA, Al-hafidh J, Barquinero JF, Lindholm C, Moquet J, Perälä M, Roch-Lefèvre S, Scherthan H, Thierens H, Vral A, Vandersickel V. 2013b. Manual versus automated gamma-H2AX foci analysis across five European laboratories: can this assay be used for rapid biodosimetry in a large scale radiation accident? Mutation Research 756:170173.

Rothkamm K, Horn S, Pope I, Barber P, Barnard S, Moquet J, Tullis I, Vojnovic B. 2012. The gamma-H2AX assay as a high throughput triage tool: Comparison of two prototype devices. NATO Science and Technology Organization Report STO-MP-HFM-223:12.

Rothkamm K, Löbrich M. 2003. Evidence for a lack of DNA double-strand break repair in human cells exposed to very low x-ray doses. Proceedings of the National Academy of Sciences USA 100:5057-5062.

Turner H, Brenner D, Chen Y, Bertucci A, Zhang J, Wang H, Lyulko O, Xu Y, Shuryak I, Schaefer J, Simaan N, Randers-Pehrson G, Yao YL, Amundson SA, Garty G. 2011. Adapting the gamma-H2AX assay for automated processing in human lymphocytes. 1. Technological aspects. Radiation Research 175:282-290.

Valente M, Voisin P, Laloi P, Roy L, Roch-Lefèvre, S. 2011. Automated gamma-H2AX focus scoring method for human lymphocytes after ionizing radiation exposure. Radiation Measurements 46:871-876. 


\section{Table 1 (on next page)}

Comparison of scoring times required for the lyse/fix and histopaque method.

Estimated time (in hours) needed for scoring 20 cells in 96 samples processed using the two different protocols . 


\begin{tabular}{lll} 
& $0 \mathrm{~Gy}$ & $1 \mathrm{~Gy}+1 \mathrm{~h}$ \\
\hline Lyse/fix & $3.1 \mathrm{~h}$ & $7 \mathrm{~h}$ \\
Histopaque & $1.5 \mathrm{~h}$ & $6.1 \mathrm{~h}$
\end{tabular}




\section{Figure 1}

\section{Sample processing times for the lyse/fix versus the routine histopaque method for different numbers of samples.}

Steps 1 - 11: 1 = lyse/fix or isolation; 2 = washing; $3=$ transfer; 4 = adhere; 5 = permeabilise (and fixation for histopaque method); 6 = blocking; $7=$ stain $1 ; 8=$ wash; $9=$ stain $2 ; 10=$ wash; $11=$ mount. Reported processing times are based on one timed experiment using 16 (lyse/fix) or 8 (histopaque method) samples and scaling estimates for the handling of 96 samples.

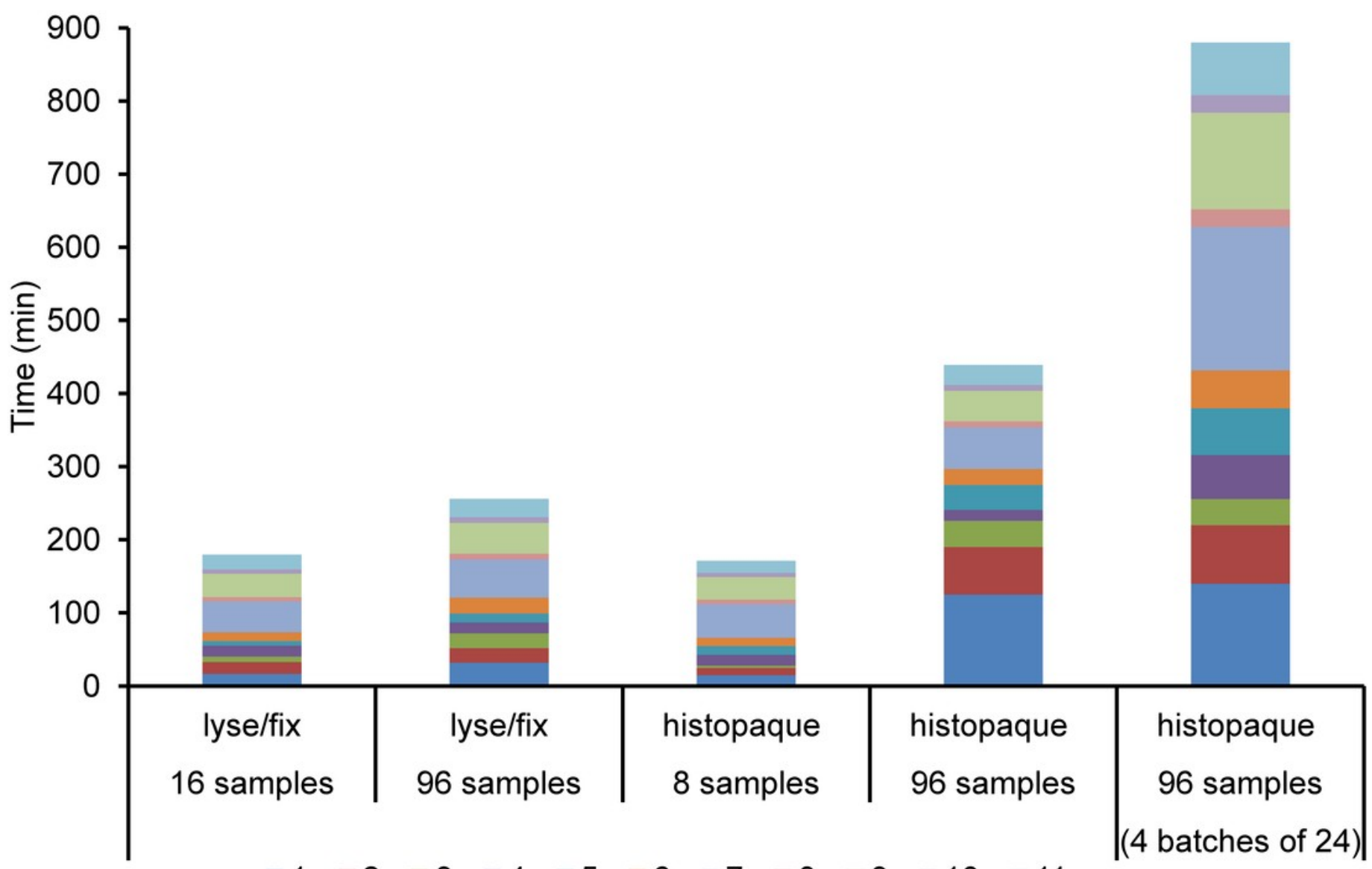

$\square 1 \square 2 \quad \square 3 \quad \square 4 \quad \square 5 \quad \square 6 \quad \square 7 \quad \square 8 \quad \square \quad \square 10 \quad \square 11$ 


\section{Figure 2}

$\gamma-\mathrm{H} 2 \mathrm{AX}$ foci in human blood leukocytes prepared with the lyse/fix or histopaque method.

Fluorescence microscopic maximum projection images ( $x 40$ objective) show $\gamma$-H2AX foci (green) and DNA counterstain (blue) following exposure to 0 or 1 Gy X-rays and incubation for 1 hour.

Lyse/fix

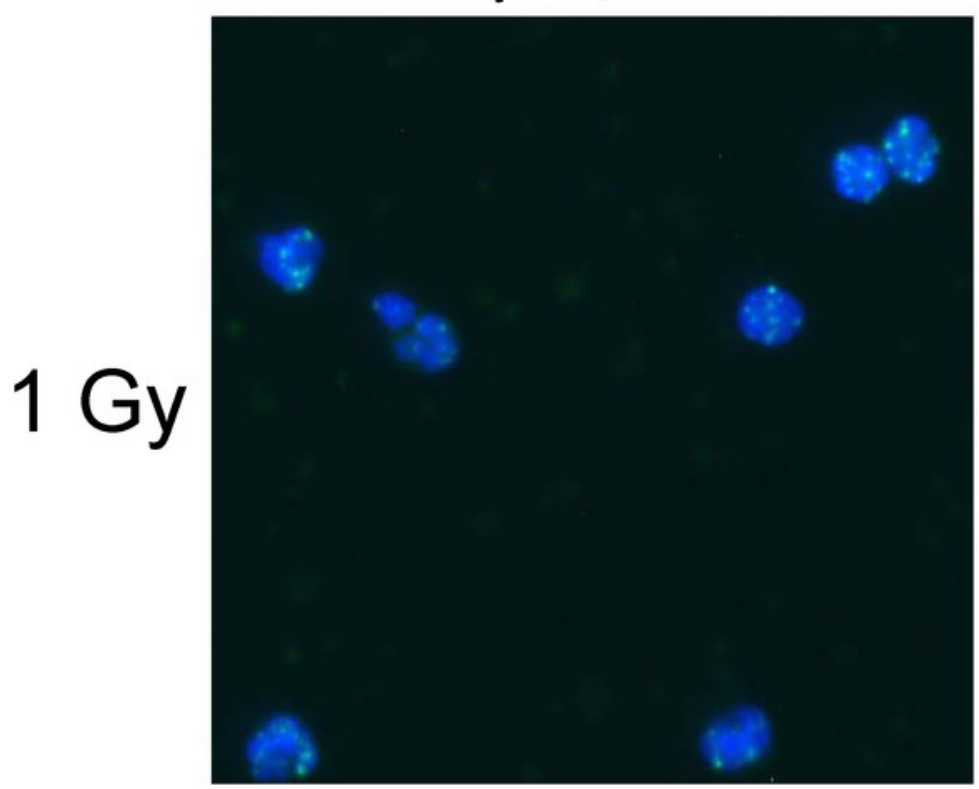

Histopaque

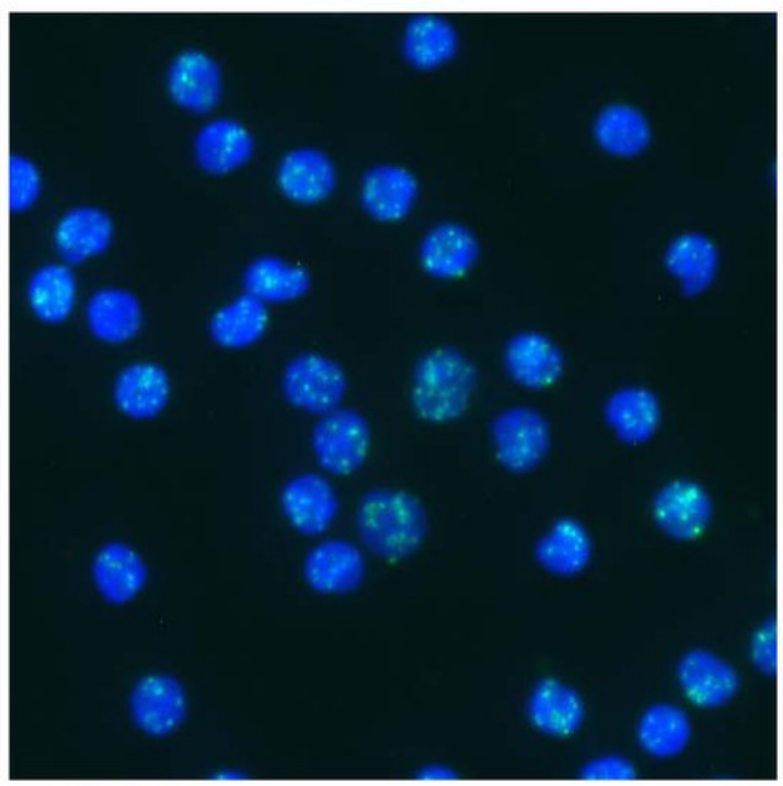

0 Gy

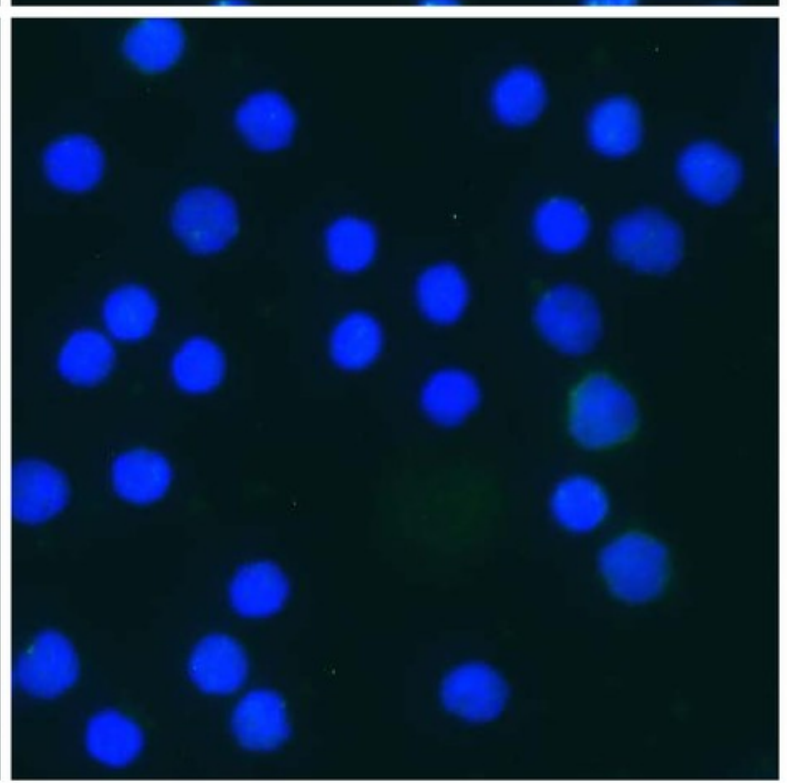




\section{Figure 3}

Comparison of manual $\gamma-\mathrm{H} 2 \mathrm{AX}$ foci counts.

Different volunteer blood samples were processed by the lyse/fix or histopaque method following 0.5 Gy X-irradiation and 30 minutes incubation at $37^{\circ} \mathrm{C}$.

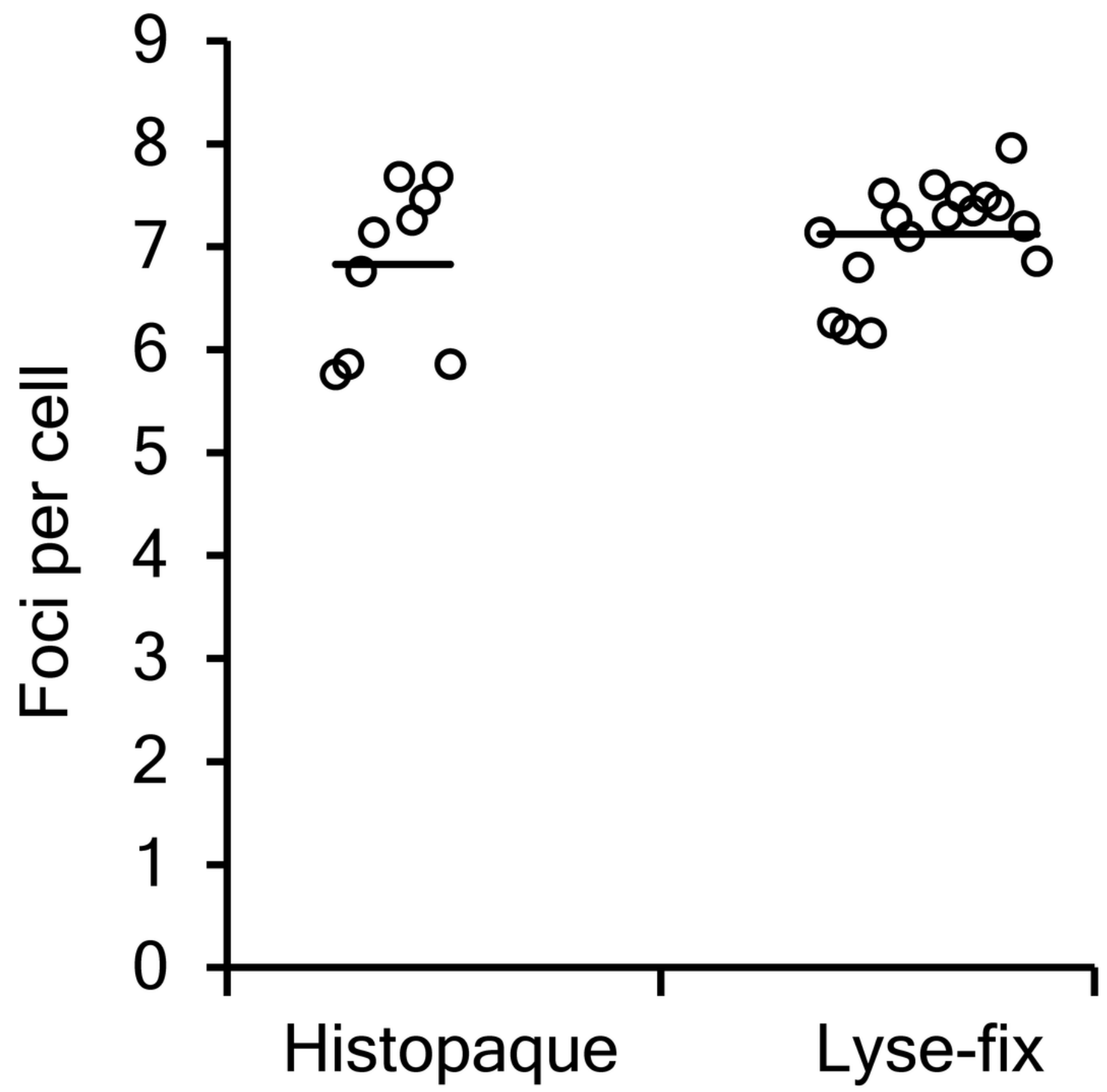




\section{Figure 4}

Comparison of automated $\gamma-\mathrm{H} 2 \mathrm{AX}$ foci counts.

Ten fields of view were analysed using Tri2 software (Barber et al, 2007) for each processing method and two irradiation conditions ( 0 or 1 Gy plus 1 hour incubation at $37^{\circ} \mathrm{C}$ ). Open triangles: 0 Gy + lyse/fix; solid triangles: 1 Gy + lyse/fix; open squares: 0 Gy + histopaque; solid squares: $1 \mathrm{~Gy}+$ histopaque.

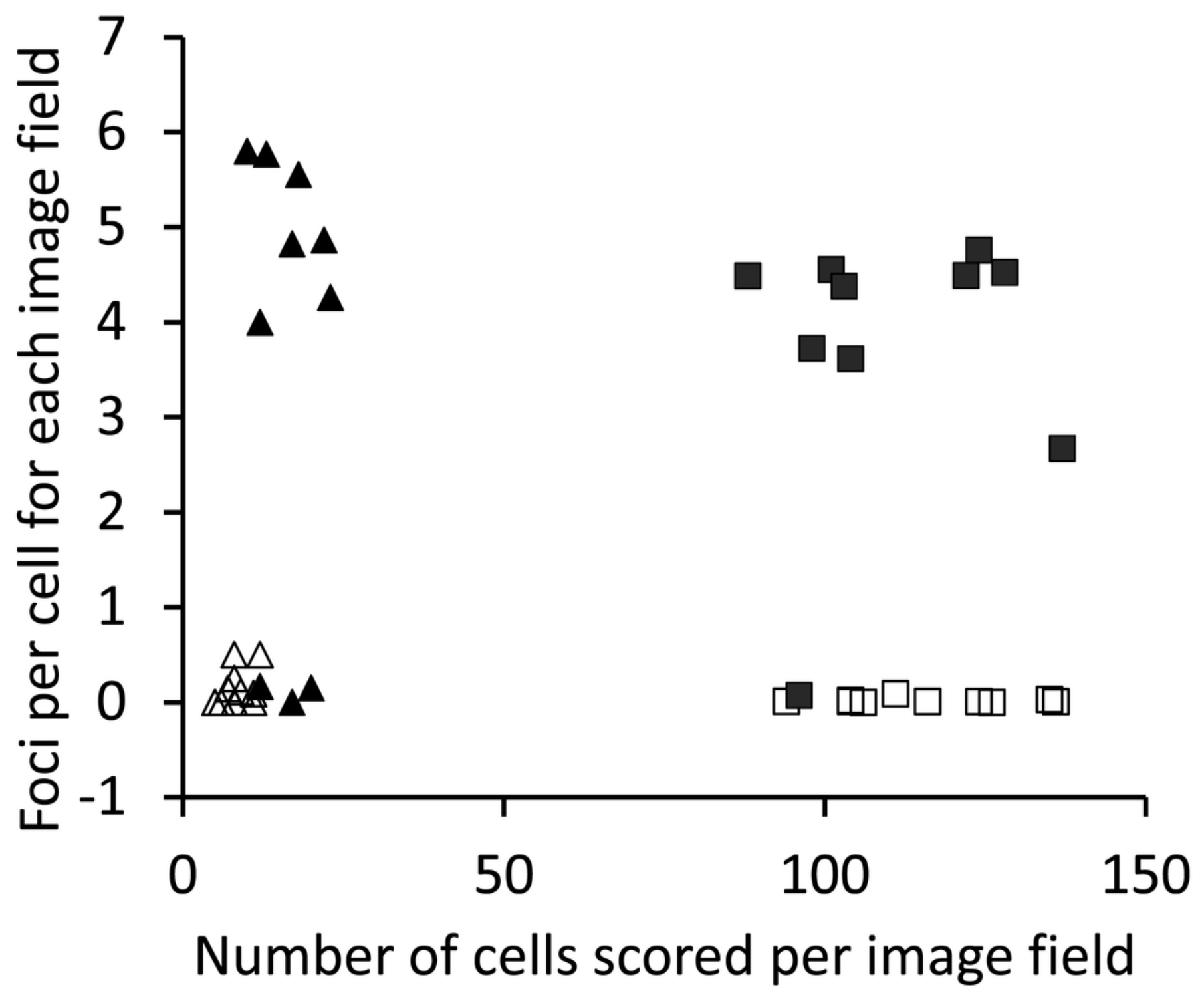




\section{Figure 5}

\section{Comparison of $\gamma-\mathrm{H} 2 \mathrm{AX}$ dose estimates.}

Foci yields were determined for $\mathrm{X}$-irradiated and 2 hours incubated blood samples processed with the lyse/fix versus histopaque protocol. At least 50 cells or 200 foci were manually scored per coded sample. Foci yields were converted to dose estimates using calibration data from Horn et al, (2011). Actual doses were 0, 0.2, 0.6, 1.1, 1.2, 2.1 and 4.3 Gy. The line indicates the ideal 1:1 relationship.

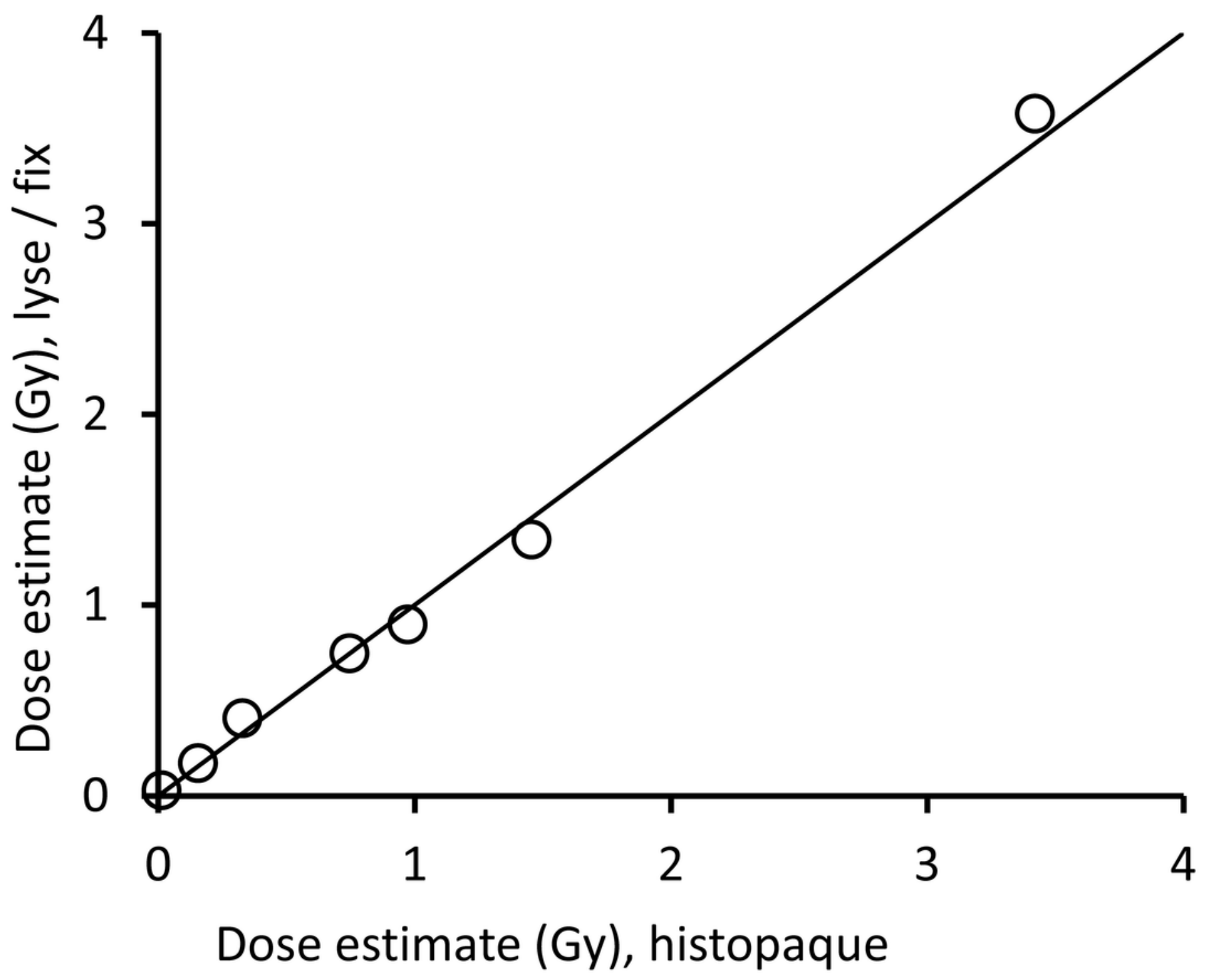

